\title{
Bérénice Zunino, Die Mobilmachung der Kinder im Ersten Weltkrieg
}

\section{Nina Régis}

\section{OpenEdition}

\section{Journals}

Édition électronique

URL : http://journals.openedition.org/ifha/10647

DOI : $10.4000 /$ ifha. 10647

ISSN : 2198-8943

\section{Éditeur}

IFRA - Institut franco-allemand (sciences historiques et sociales)

\section{Référence électronique}

Nina Régis, «Bérénice Zunino, Die Mobilmachung der Kinder im Ersten Weltkrieg », Revue de l'IFHA [En ligne], Date de recension, mis en ligne le 28 mars 2020, consulté le 24 septembre 2020. URL : http:// journals.openedition.org/ifha/10647 ; DOI : https://doi.org/10.4000/ifha.10647

Ce document a été généré automatiquement le 24 septembre 2020

(CIFHA 


\title{
Bérénice Zunino, Die Mobilmachung der Kinder im Ersten Weltkrieg
}

\author{
Nina Régis
}

\section{RÉFÉRENCE}

Bérénice Zunino, Die Mobilmachung der Kinder im Ersten Weltkrieg. Kriegskultur und illustrierte Kriegskinderliteratur im Deutschen Kaiserreich (1911-1918), Berlin : Peter Lang, $2019,56,10 €$ 
L'ouvrage de Bérénice Zunino sur la mobilisation des enfants pendant la Grande Guerre à travers la littérature illustrée est le fruit d'une thèse en cotutelle entre Paris Sorbonne et la Freie Universität de Berlin sous la direction de Jean-Paul Cahn et d'Oliver Janz. Il s'inscrit dans un contexte académique et historiographique français dans lequel deux écoles se font face, dites de "Péronne» et de "Craonne», cristallisées autour des débats sur les motivations qui poussaient les soldats à poursuivre le combat, par le consentement ou par la contrainte. Sans se positionner, l'ouvrage mobilise ces concepts (p. 30 à 32), ainsi que ceux de la "brutalisation secondaire et tertiaire"
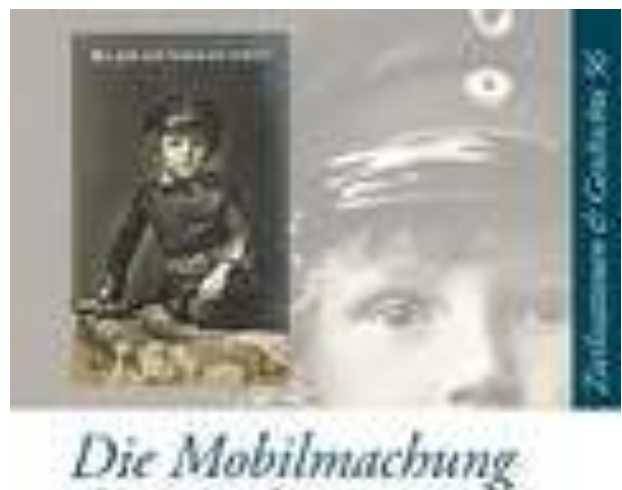
der Kinder im Ensten Weltkrieg

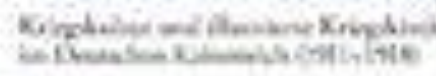
Wiesterese


(p. 35) et cherche à comprendre en quoi ce conflit marqua le début d'une « culture de guerre ", distincte des traditions iconographiques allemandes plus anciennes en étudiant le " degré de rupture » entre les images produites avant et pendant la guerre. L'ouvrage innove à travers des chiffres de tirage inconnus, l'analyse pointue du manque de succès de la littérature illustrée auprès des enfants, l'analyse minutieuse d'aspects linguistiques et discursifs, et repose sur l'exploitation de nombreux fonds d'archives, de bibliothèques et de musées allemands.

Récemment, l'ouvrage a suscité l'intérêt d'Eberhard Demm qui en a rédigé un compterendu en allemand. Bien que certains reproches ayant été adressés par cet historien à l'ouvrage soient justifiés, d'autres restent trop vagues pour être vérifiés, notamment celui du quasi-plagiat, culminant dans une négation de toute innovation scientifique, qu'il contredit par ailleurs. Selon le chercheur, l'étude ignore la littérature secondaire, et en particulier deux de ses articles. En revanche, cet oubli ne peut justifier l'omission des apports de l'ensemble de l'appareil critique et bibliographique mobilisé. Le fait que celui-ci soit issu d'une thèse effectuée dans un contexte transnational, publié en allemand, représente d'emblée un apport indéniable à la recherche, demandant des capacités et des efforts auxquels tout chercheur, qu'il soit jeune ou confirmé, n'est pas toujours prêt à se confronter.

Divisé en quatre chapitres, cet ouvrage revient d'abord sur les origines des illustrations dans la littérature pour enfants avant 1914. Dans un second temps, l'auteure étudie l'entrée progressive de la guerre au sein de l'iconographie, puis se tourne vers la mobilisation des enfants et des représentations idéalisées. Le dernier chapitre analyse leur mobilisation à travers cette littérature illustrée. La conclusion est la suivante : en 1914, loin de marquer une rupture, la littérature pour enfants puise encore dans le répertoire iconographique antérieur à la Grande Guerre. En décelant une continuité entre les représentations du début de la guerre avec la tradition iconographique, l'ouvrage approfondit les études portant sur les discours de propagande adressés aux enfants pendant la Grande Guerre. Les éléments nouveaux, tels que l'incitation à 
participer aux collectes de faines, de noyaux de fruits, de cheveux ou encore d'objets en fer, ainsi que l'hérö̈sation des aviateurs, n'apparaissent en réalité que progressivement. B. Zunino remarque une différence importante avec l'iconographie enfantine en France : dans l'Empire, les pédagogues étaient attachés à ne pas brusquer les jeunes esprits, tout en les encourageant à se mobiliser, à prendre des responsabilités et à faire des sacrifices pour la Patrie. Sur un autre plan, l'étude rejoint celles de Stéphane Audoin-Rouzeau, d'Annette Becker, de Christian Ingrao et d'Henry Rousso ( $L a$ violence de guerre, 1914-1945, 2002) ainsi que celle d'Arndt Weinrich (Der Weltkrieg als Erzieher, 2012). Elle s'emploie à identifier les racines des parcours des futurs nationauxsocialistes en reconstruisant les coulisses iconographiques du temps de leur enfance. L'ouvrage contribue ainsi aux analyses menées dans un champ de recherches florissant, à savoir celui de l'enfance en temps de guerre.

Les liens étroits entre la performativité des images, l'imaginaire et la perception du réel dans l'enfance (p.26) se situent au premier plan de l'analyse et jouent un rôle fondamental en tant que piliers sur lesquels les individus reviennent constamment puis continuent à bâtir, plus ou moins consciemment. À travers la relation père-enfant (p. 179), la mobilisation des sentiments (p. 189), l'appel aux affects enfantins à travers l'image, où le rire sert d'échappatoire aux maux du quotidien (p.124), apparait en filigrane une histoire des émotions et des sensibilités. On aurait, de la même manière, pu mettre en avant une histoire du genre car la culture de guerre adressée aux enfants reflète et intensifie le discours de la répartition traditionnelle des rôles (p. 201) assignés aux femmes, "mères aimantes et femmes fidèles» (p.152), et aux hommes à travers "[l'] idéal d'une masculinité forte» (p.180). Cette démarche suppose de nombreux défis méthodologiques, dont celui de la représentativité, « la plus grande difficulté à laquelle les historiens font face " selon Christophe Prochasson. Il est en effet très probable que le public visé ait inclut les parents par le biais de leurs enfants. En revanche, l'auteure le souligne, l'analyse donne un aperçu de l'univers culturel et discursif des enfants issus de milieux aisés, en mesure d'acquérir cette littérature illustrée. À partir de 1917, lorsque le papier devient onéreux, les prix augmentent et la clientèle se raréfie, la production s'effondre. Bien que l'étude se restreigne à cette couche privilégiée, elle prend tout son sens dans la mesure où de nombreux dirigeants nazis étaient issus de ces mêmes milieux. En osant poser cette question difficile mais pertinente, ce travail contribue ainsi à la compréhension des pierres angulaires des parcours de vie des grands acteurs du second conflit mondial.

Cependant, la réception effective demeure incertaine, notamment quant au degré et à l'étendue de la performativité des images étudiées. Puisque leur diffusion cesse bien avant la fin de la guerre, l'impact de cette littérature a peut-être été surestimé, dans un contexte où des pénuries et l'inflation touchaient jusqu'aux familles de la bourgeoisie intellectuelle, comme celle du professeur d'université Karl Hampe ou celle de Friedrich Meinecke, susceptibles d'acheter ces livres. Une analyse plus poussée d'un des parcours menant à une adhésion aux idéaux et au parti national-socialiste, qui ne pouvait être menée dans le cadre de cette étude, permettrait de donner un exemple plus concret de ce type d'évolution individuelle, marquant une continuité entre le premier et le second conflit mondial. Tout comme dans le dernier livre de Gerd Krumeich (Die unbewältigte Niederlage: das Trauma des Ersten Weltkriegs und die Weimarer Republik, 2018), le journal de Jo Mihaly (Elfriede Kuhr) illustre bien le propos de l'auteur (p. 169). L'exploration d'un corpus de journaux intimes aurait également pu donner des indices sur la réception des images dans la littérature pour enfants. En outre, l'argumentation mobilise les concepts 
d'agency (p.192), de performativité des images ainsi que d'acte d'image (Bildakt) et indique par conséquent que ces représentations iconographiques guident l'action. L'impact de ces illustrations sur l'engagement concret des enfants pour leur patrie reste tout de même difficile à démontrer à grande échelle. Des recherches complémentaires sur les enfants issus des milieux aisés seraient en mesure de consolider cette argumentation. Quelques questions qui pourraient donner lieu à des développements ultérieurs restent en suspens : pourquoi les pédagogues continuèrentils à conseiller les livres patriotiques pour enfants jusqu'à la fin de la guerre et comment se fait-il qu'ils continuèrent à être édités en dépit de l'inflation et de la grande rareté du papier ? Croyaient-ils encore en « la victoire décisive» (p. 233) ? Cela paraît peu probable car dès la fin de l'année 1915, les grèves, les soulèvements et les témoignages montrent qu'une très large majorité de la population ne croit plus à la victoire. Au vu des pénuries alimentaires, de l'inflation et de la dégradation des conditions de vie, les images héroïques permettaient-elles aux enfants de se distraire des réalités quotidiennes (p.143) ? Pourrait-on supposer que ces images eurent l'effet inverse sur des jeunes gens trop tôt devenus adultes, devant remplacer les pères et soutenir les mères?

En tout état de cause, outre d'approfondir et d'apporter un éclairage nouveau sur les évolutions, les continuités et les innovations dans l'iconographie destinée aux enfants qui, en tant qu'adultes, furent acteurs de la Seconde Guerre mondiale, l'ouvrage soulève de nouvelles questions et ouvre la voie à de nouveaux champs d'études liés à l'enfance pendant la Grande Guerre. L'auteure de cette étude mérite toute la reconnaissance de ses pairs pour son travail de recherche considérable à de très nombreux égards. Contrairement aux recherches précédentes sur la littérature et l'iconographie destinée aux enfants, elle met l'accent sur l'impact potentiel de ces publications sur les acteurs de la Seconde Guerre mondiale. Bien que des recherches plus approfondies doivent être menées pour confirmer cette analyse, B. Zunino ne tombe pas dans le travers que constituerait l'analyse superficielle d'une documentation abondante et massive, et mène l'étude rigoureuse d'une sélection de sources judicieusement insérées dans un plan et une argumentation clairs. Par conséquent, l'ouvrage en question apporte une contribution notable à la recherche collective portant sur la Grande Guerre, l'histoire de l'enfance et celle des représentations.

INDEX

Index chronologique : Époque contemporaine

Thèmes : Histoire des mentalités ; Histoire sociale

\section{AUTEURS}

NINA RÉGIS

Université Toulouse Jean-Jaurès / DHIP/IHA Paris 